\title{
A neurally-interfaced hand prosthesis tuned inter-hemispheric communication
}

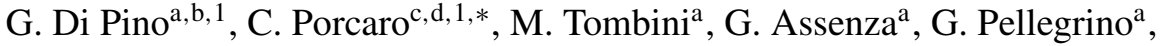 \\ F. Tecchio ${ }^{\mathrm{c}, \mathrm{e}, 2}$ and P.M. Rossini ${ }^{\mathrm{e}, \mathrm{f}, 2}$ \\ ${ }^{a}$ Department of Neurology, Campus Bio-Medico University, Rome, Italy \\ ${ }^{\mathrm{b}}$ Biomedical Robotics and Biomicrosystems Lab., University Campus Bio-Medico, Rome, Italy \\ ${ }^{\mathrm{c}}$ LET's - ISTC - CNR, Ospedale Fatebenefratelli, Isola Tiberina, Rome, Italy \\ ${ }^{\mathrm{d}}$ Institute of Neuroscience, Newcastle University, Medical School, Newcastle upon Tyne, UK \\ ${ }^{\mathrm{e}}$ Casa di Cura S. Raffaele, Cassino and IRCCS S. Raffaele-Pisana, Rome, Italy \\ ${ }^{\mathrm{f}}$ Institute of Neurology, Catholic University, Rome, Italy
}

\begin{abstract}
Purpose: This work investigates how a direct bidirectional connection between brain and hand prosthesis modifies the bi-hemispheric sensorimotor system devoted to the movement control of the lost limb. Hand prostheses are often unable to satisfy users' expectations, mostly due to the poor performance of their interfacing system. Neural Interfaces implanted inside nerves of the stump offer the advantage of using the bidirectional neural pathways 'naturally' dispatching signals to control proper hand actions and feed-back sensations. Learning to control a neurally-interfaced hand prosthesis and decode sensory information was previously observed to reduce the inter-hemispheric asymmetry of cortical motor maps and the clinical symptoms of phantom limb syndrome.

Methods: Electroencephalographic (EEG) data was analysed using Functional Source Separation (FSS), a semi-blind method that incorporates prior knowledge about the signal of interest into data decomposition to give access to cortical patch activities. Results: Bi-hemispheric cortices showed normalization of their activity (topographical and spectral patterns) and of functional connectivity between homologous hand controlling areas, during the delivery of the motor command to the cybernetic prosthesis. Conclusions: The re-establishment of central-peripheral communication with the lost limb induced by a neurally-interfaced hand prosthesis produces beneficial plastic reorganization, not only restructuring contralateral directly-connected control areas, but also their functional balance within the bi-hemispheric system necessary for motor control.
\end{abstract}

Keywords: Functional source separation, hand prosthesis, neural interface, inter-hemispheric coherence, neurorehabilitation

\section{Introduction}

Human beings are able to interact with the environment through several modalities, which involves neural signals passing through the nerves, muscle activity

\footnotetext{
${ }^{1}$ These authors contributed equally to this work as first author.

${ }^{2}$ These authors contributed equally to this work as last author.

*Corresponding author: Dr. Camillo Porcaro, LET'S-ISTCCNR, Department of Clinical Neuroscience, Fatebenefratelli Hospital - Isola Tiberina, 00186 Rome, Italy. E-mail: camillo.porcaro@istc.cnr.it.
}

and the integrity of cutaneous/proprioceptive sensory organs. Brain Machine Interfaces (BMI) bypass the above steps and allow motor signals to be picked up directly from the nervous system and relayed to an external electronic or robotic effector which physically performs the subject's motor intention and plans. In addition to facilitating extraction of motor volition, bidirectional BMIs also create the capacity to manage neural signal flows in both afferent and efferent directions.

Hand/finger manipulation is a very complex activity performed almost exclusively by humans and a 
few primates. Although prosthetics was one of the first applications for artificial human-like hands, engineers and roboticists that developed multi-fingered humanoid robots and mechatronic hand prostheses, have not so far been able to achieve the same manipulative ability owned by the human hand (Carrozza et al., 2006; Zollo et al., 2007). However, many efforts with significant progresses have been achieved in this direction.

To date, what the market is able to offer to upper limb amputees still suffers a series of limitations related to their mechanics, control system and embedded proprioceptive and exteroceptive sensors. However, a fundamental limitation afflicting their performance is the poverty of the user-device interplay achievable by present interfacing systems (Micera et al., 2006). Hence, the control of anthropomorphic hand prostheses represents a promising field for the application of neural interfaces.

At the amputation site all afferent and efferent nerves, originally devoted to the lost segment, are interrupted. The consequent deprivation of peripheral inputs and actuators results in retrograde changes that affect not only the peripheral nervous system but also central structures including the motor and somatosensory cortices. In fact, following amputation the deafferented cortical areas become responsive to inputs from the parts of the body that are represented adjacent in the Penfield homunculus (Kaas, 1991; Merzenich et al., 1984; Pons et al., 1991; Ramachandran et al., 1992).

While a wide literature indicates the adaptive potential of brain plasticity, mainly in conditions of congenital deficits (for reviews on blind people see Pascual-Leone et al., 2005; Sadato, 2005, Gilbert and Walsh, 2004), experience in amputee suggests that the traumatic event prevents adaptive cortical changes. In fact, the aberrant counterpart of plastic phenomena probably represents the pathophysiological substrate of a disabling and painful experience called phantom limb pain (PLP), that afflicts the majority of amputees and is widely considered as a maladaptive correlate of neuroplasticity (Flor et al., 1995; Flor et al., 2006). Therefore it seems reasonable to assume that the amount and characteristics of PLP are some of the indicators of harmful cortical reorganization. For a comprehensive review of neuroplasticity in amputees see (Di Pino et al., 2009).

Commonly the ability possessed by the majority of amputees to move the phantom of the lost limb at their will was considered as motor imagery. Recently it has been shown that after amputation the ability to execute a movement with the affected limb is maintained by the amputees' brain (Raffin et al., 2011), despite the absence of a peripheral effector. In fact motor imagination and execution differentially activate cerebral areas with a significantly greater activation in primary motor and sensory cortices during execution, while imagination is associated with greater parietal and occipital lobe activity (Raffin et al., 2012). Even though the nervous system is altered by the amputation, residual neural patterns appropriate for the lost limb may still be activated when suitably stimulated (Mercier et al., 2006; Reilly et al., 2006). Those pathways are therefore a possible target of central or peripheral neural implants to restore a direct and relatively 'natural' channel for data exchanging.

Multielectrode arrays implanted inside peripheral nerves represent an optimal compromise between the selectivity of exchanged data and the invasiveness of the required surgery. Their fundamental ability to interact with a small number of nerve fascicles and to exchange information bidirectionally (Micera and Navarro, 2009; Micera et al., 2008; Navarro et al., 2005) make such interface appropriate to enhance neuroplastic phenomena (Di Pino et al., 2009).

A positive correlation between use of a nerveinterfaced hand prosthesis and the reduction of aberrant brain plasticity has already been described by our group using TMS cortical mapping and PLP reduction assessment (Rossini et al., 2010), and by analysis of event-related EEG synchronization/desynchronization (Tombini et al., 2012).

The present study explores whether and how a direct connection between the brain and a hand prosthesis via a neural implant modifies bi-hemispheric EEG activity in primary sensorimotor cortical areas controlling movements of the lost limb. In particular we apply a Functional Sources Separation (FSS) comparative analysis of the EEG pattern recorded during the intent of voluntary moving the lost limb phantom, collected before and after the implant period.

EEG and MEG signals are linear mixtures of source activities, and are often analysed using the blind source separation (BSS) technique. These algorithms estimate the complete source time-courses based on statistical properties of the generated signal, without taking into account the physical nature of the generating phenomenon. Thus, BSS procedures use only information contained in the waveform of original 
signals, a convenient property for neurophysiological techniques (EEG and MEG) which provide the most informative time-frequency signal from the intact human brain. BSS techniques, in particular independent component analysis (ICA) algorithms, have been successfully applied to EEG and MEG data (Barbati et al., 2004; Makeig et al., 2004; Medaglia et al., 2009; Porcaro et al., 2011; Porcaro et al., 2006). The aim of such techniques is to decompose in a 'blind' fashion (i.e., without making specific assumptions) the sources underlying the EEG and MEG recorded signals (for a comprehensive review on BSS see: Hyvarinen et al., 2001; Cichocki and Amari, 2002; Tecchio et al., 2007b). Our group developed a new semi-BSS method called Functional Source Separation (FSS) (Barbati et al., 2006; Tecchio et al., 2007b), an extension of ICA that incorporates prior information into the decomposition, resulting in a single component which contains a hallmark of the source of interest (for this reason it has been denominated semi blind method). The aim of FSS is to enhance the separation of relevant signals by exploiting some a priori knowledge (i.e., spectral characteristics or evoked activity property) without losing the advantages of using only information contained in original signal waveforms. In other terms, FSS allows the identification and analysis of specific neuronal pools on the bases of their functional properties instead and independently of their spatial position. This is an extremely helpful feature especially in those cases where cerebral plastic changes have altered the location of brain functions with respect to standard anatomical landmarks typical of healthy people.

\section{Methods}

\subsection{Subject and prosthesis control}

A brief description is given in the following section, while a complete report on the clinical and technical aspects of this case can be found elsewhere (Rossini et al., 2010).

A 26 year-old right-handed male (PP) was recruited two years after a left arm trans-radial amputation. His previous medical history was unremarkable. The study was approved by the local Ethics Committee and the assigned office of Italian Ministry of Health, and an informed consent was signed by the patient. P.P. referred to a moderate phantom limb syndrome and perceived his phantom limb as if '...the missing hand is still attached to the stump and tightly fastened by a belt without any forearm'.

The subject had been implanted with four longitudinal intra-fascicular electrode arrays (tf-LIFE4s) (Hoffmann and Kock, 2005) in the Ulnar and Median nerves of his stump for four weeks, providing a total amount of 32 bidirectional contacts.

Before the implantation the subject was trained for two weeks in dispatching the motor commands to perform movements with his phantom hand, without activating stump muscles.

Three movements of a virtual left hand were shown in videos: power grip, pinch grip and flexion of the little finger. The actions were exploited because they represent the range of movements controlled by the implanted nerves: median fibres for the pinch, ulnar for little finger flexion, and both for the power grip.

During the four experiment weeks after electrode implantation, PP was required to perform the hand grip tasks triggered by the same videos, while recording neural signals.

Signals from tf-LIFE4s, biceps and triceps EMG electrodes were simultaneously recorded using a $48 \mathrm{kHz}$ sampling rate. The mean, rectified signals were calculated for bins of width 1000 samples.

Each prosthesis movement type was triggered by the signal level of a single channel with the best signal-to-noise ratio. Channels were coupled with grips depending on their anatomo-functional location (i.e., channels from the median nerve for power or pinch grip, channels from the ulnar for little finger flexion). To avoid interference from muscle activity or environmental noise, only rectified values greater than $3 \mu \mathrm{V}$ in a time window ranging from 5 to $20 \mathrm{~ms}$ were used.

Together with visual feedback from the prosthesis posed in front of him, PP received sensory feedback via suprathreshold electrical pulses delivered through the implanted electrodes, triggered by the experimenter (Fig. 1). Stimulus trains $(0.3 \mathrm{~s}$ train of $70 \mathrm{~Hz}, 10 \mu \mathrm{A}$, $10 \mu$ s pulses) were delivered after each burst of efferent activity recorded by tf-LIFE4 $\mathrm{s}$. The channels used to select when and to provide the stimulation were the same, and were established according to the performed action (i.e., channels into the median nerve for power or pinch grip, channels into the ulnar for little finger flexion, channels into both nerves for the power grip). In all cases, the subject described discrete tactile (nonproprioceptive) sensations in the fascicular projection territories that corresponded to the stimulated nerves. 


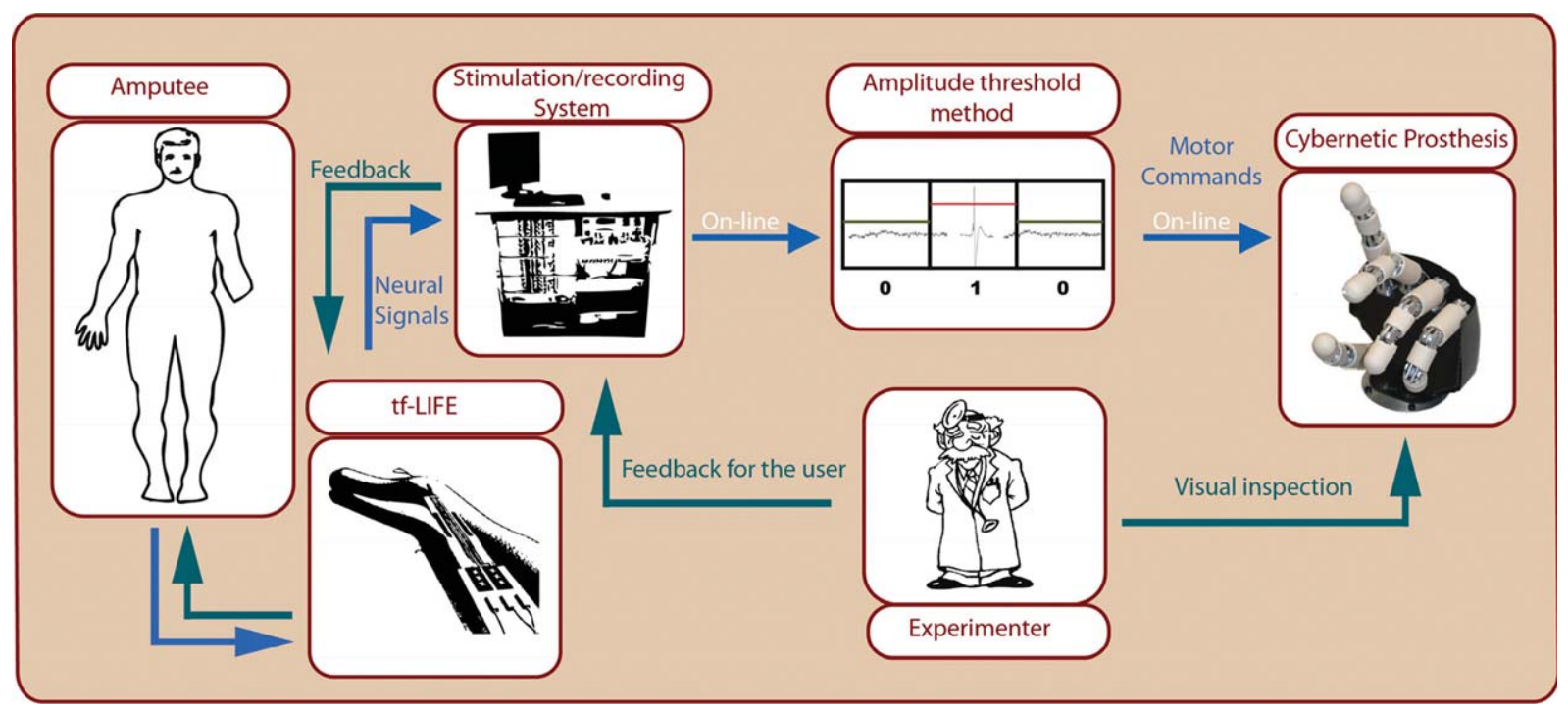

Fig. 1. Block scheme of the online control of the prosthetic device.

\subsection{EEG recordings}

EEG signals were recorded from the scalp during a handgrip task including periods lasting 5 seconds of active control of motor commands dispatched to the hand (movement), intermingled with $5 \mathrm{~s}$ periods of relaxation (recovery). The study of left phantom hand movement was executed at two times: i) after pre-implant training the day before surgery (PRE), ii) at the end of four weeks of intensive training to control the hand prosthesis using implanted LIFEs (POST). Thirty-two electrodes (scalp sites defined according to the international 10-20 electrode system) were mounted on an elastic cap and a binaural reference was used. Skin/electrode impedances were kept below $5 \mathrm{k} \Omega$. Recordings were carried out using a time constant of $0.1 \mathrm{~s}$. EEG data were sampled at $1024 \mathrm{~Hz}$ (pre-sampling analogical filter $0.48-256 \mathrm{~Hz}$, BrainAmp System).

\subsection{Data analysis}

The first step of the analysis we have employed is a semiautomatic independent component analysis (ICA)-based procedure to identify and remove cardiac and/or ocular artifacts without rejecting the contaminated epochs (Barbati et al., 2004).

The functional source separation (FSS) procedure (Porcaro et al., 2009; Porcaro et al., 2010; Tecchio et al., 2007b) was applied to identify the cortical neural networks devoted to hand movement. As in the ICA approach, FSS starts from an additive hidden source model of the type $x=A s$, where $x$ represents the observed EEG data, $s$ are the underlying unknown sources and $A$ is the source-sensor coupling matrix to be estimated. Additional information to a standard ICA model is used to bias the decomposition algorithm towards solutions that satisfy physiological assumptions. A modified (with respect to standard ICA) contrast function is defined:

$$
F=J+\lambda R_{F S}
$$

where $J$ is the statistical index normally used in ICA and $\mathrm{R}_{\mathrm{FS}}$ accounts for the a-priori information exploited to identify the source. Using the weighting parameter $\lambda$ it is possible to adjust the relative weight of these two aspects. In this study, $\lambda$ was chosen to be equal to 1000 in all cases, as detailed in Porcaro and colleagues (Porcaro et al., 2008). Briefly, $\lambda$ was chosen to both minimize computational time and maximize $R_{F S}$. This scheme allows the possibility of extracting only one component that maximizes the functional behavior in agreement with the functional constraint. In the present work, a maximization of the difference in Power (Power Spectral Density, PSD) between rest and task was used. 


\subsection{Primary sensorimotor functional source (FS_hand)}

The functional constraint exploited mu rhythm (8-25 Hz) reactivity (Pfurtscheller and Lopes da Silva, 1999) that occurs in contralateral sensorimotor areas during uni-manual motor tasks, by requiring maximal variation of spectral power in alpha and beta bands between the period of prosthesis control and rest. The ad-hoc functional constraint $R$ was thus as follows:

$$
R\left(F S_{\alpha+\beta}\right)=\frac{\sum_{\alpha+\beta} \operatorname{PSD}\left(F S_{\alpha+\beta}\right)_{\text {Task }}-\sum_{\alpha+\beta} \operatorname{PSD}\left(F S_{\alpha+\beta}\right)_{\text {Rest }}}{\sum_{\alpha+\beta} P S D\left(F S_{\alpha+\beta}\right)_{\text {Rest }}}
$$

with the Power Spectrum Density (PSD) during Task estimated in the $5 \mathrm{sec}$ windows of each movement trial and at Rest in the $5 \mathrm{sec}$ windows preceding each trial. $\alpha+\beta$ frequency band included 8 to $25 \mathrm{~Hz}$ (Barbati et al., 2008; Porcaro et al., 2011).

\subsection{Functional source behavior}

Cortical activity of areas devoted to hand control was evaluated by time-frequency modulations of FS power spectral density during the motor task as compared to the resting state baseline. Twenty single trials from the source were chosen in the time interval of -5 to $5 \mathrm{~s}$, where time 0 is the movement go signal. They were convolved by a Morlet wavelet (setting equal to 7 the constant which defines the compromise between time and frequency resolution), and the squared absolute values of the convolution over trials were averaged. For each frequency, the time course of power modulation was represented as the percentage of the mean of the baseline period ( -5 to $0 \mathrm{~s}$ ). The cortical recruitments and the time frequency behavior were compared before and after neural implant.

Significant power changes from Rest to Movement Imagination (Task) periods were assessed using a resampling bootstrap technique thresholded at $P=0.05$, while non-significant changes were set to 0 . The above procedure was applied for the PRE, POST and on the right hand movement.

\subsection{Functional source localization}

In order to investigate cortical recruitment occurring during the intention of moving of the (intact) right hand and (phantom-prosthetic) left hand before
(PRE) and after training with the implanted neural electrodes (POST), the FSs identified by FSS were submitted to a source localization algorithm (sLORETA - (Pascual-Marqui, 2002)) implemented in CURRY 6 (Neuroscan, Hamburg, Germany, http://www.neuroscan.com/). sLORETA was performed for each source using a regular grid with a spacing of $3 \mathrm{~mm}$ throughout the brain region and a four shell spherical head model. The results were projected onto the template brain of the Montreal Neurological Institute (MNI) within CURRY.

\subsection{Functional connectivity between homologous hand control areas}

The functional connectivity between the hemispheric homologous primary cortical circuits was estimated by spectral coherence between the activities of the areas devoted to the control of the contralateral hand. Instead of anatomical criteria, we preferred to select the region on a functional basis, thus we considered the areas activated during the right and left motor tasks. Being interested in primary circuits, in the case of the left affected hand we chose the region of interest (RoI) corresponding to the FS in the POST session, i.e., the primary contralateral right RoI, since the FS in the PRE-session involved a bilateral network prevailing in the ipsi-lateral left sensorimotor areas. A standard FFT approach (Welch technique and Hanning windowing) was applied in the same time windows used for PSD estimates:

$$
\left|C_{x y}(f)\right|^{2}=\frac{\left|S_{x y}(f)\right|^{2}}{S_{x}(f) S_{y}(f)}
$$

with $f$ the frequency bin, $x$ and $y$ the $F S_{\text {_right hand }}$ and FS_left hand, $S_{x y}$ the cross spectrum between $x$ and $y, S_{x}$ and $S_{y}$ the spectra of $x$ and $y$ respectively. The modulus of coherence takes a dimensional values in the $[0,1]$ range (0 indicates no relationship). The $95 \%$ confidence limit is estimated according to (Halliday et al., 1995) by $(1-0.05)^{\frac{1}{(L-1)}}$, where $L$ is the number of trials used to estimate the coherence function. 


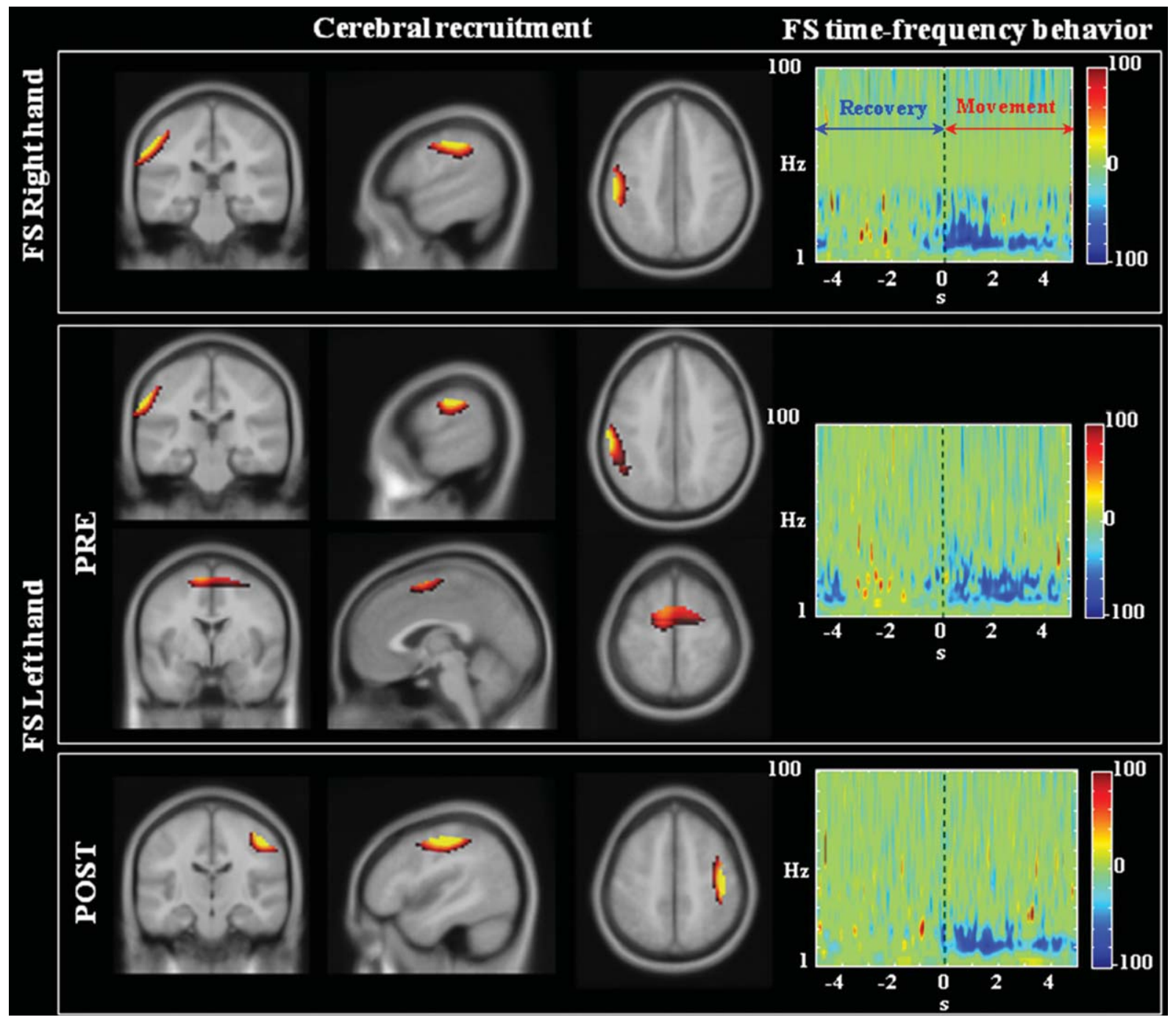

Fig. 2. FSs' cerebral recruitment and time-frequency behavior. From Left: solutions of the sLORETA model localization algorithm for the FSs related to right hand control (Up Left), to the missing limb phantom control before LIFE implant (PRE - Middle Left) and at the end of 4 weeks-long training with LIFEs implanted (POST - Bottom left) are shown superimposed on the MNI brain template in coronal, sagittal and axial sections. Right: Time frequency representation of each FS activity, 0 correspond to the motor Task initiation. The color code represents significant changes in average power (across epochs) as a function of time and frequency. Values for a given frequency bin are expressed as percentage of the Rest period. Power change significance was assessed by using a bootstrap technique and thresholded at $p=0.05$; non-significant

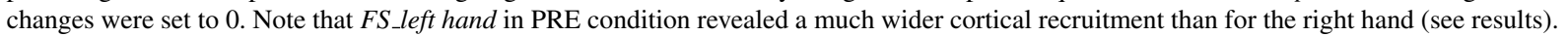

\section{Results}

\subsection{Movement of the right hand}

Right hand movement recruited well segregated contra-lateral left sensorimotor areas (Fig. 2 - first row). In particular, source localization shows a clear activation of the primary sensory and motor areas (left Postcentral Gyrus - BA2, BA3 and Precentral Gyrus -
BA4). The analysis of time frequency behavior exhibited a clear response that was stronger in the first 2 seconds and covered the whole mu band including both alpha and beta frequencies, becoming concentrated in the alpha band for the $2-5 \mathrm{~s}$ period.

Cortico-cortical coherence of EEG rhythms between the homologous primary areas devoted the right and left hand motor control (Fig. 3) displayed a clear prevalence in alpha and beta frequency ranges during both 

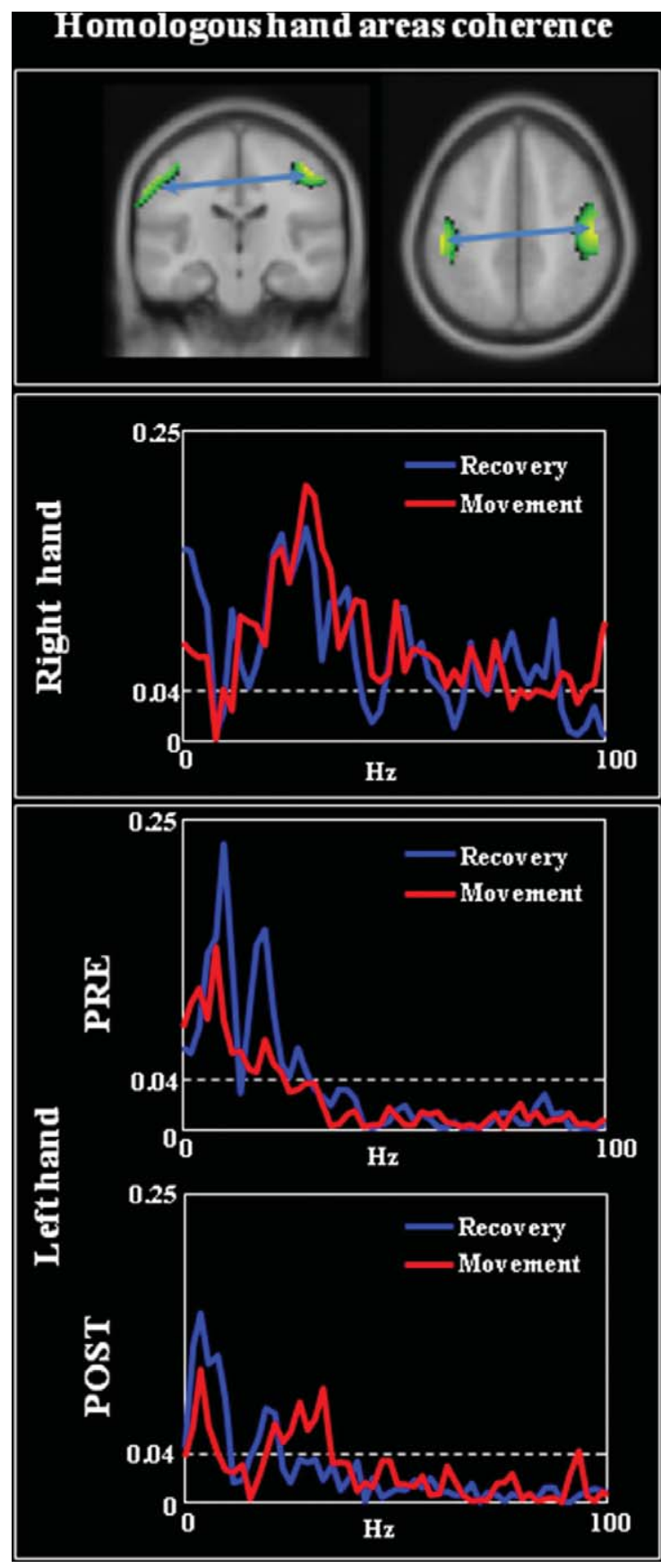

Fig. 3. Homologous hand areas' coherence. Top: solutions of the sLORETA model localization algorithm (coronal and sagittal view) for the cerebral recruited hand areas (see methods) superimposed on the MNI brain template. Bottom: Cortico-cortical coherence between the cerebral recruited areas during recovery (blue line) and movement (red line) periods. Horizontal line indicates the confidence limit (see Materials and Methods section). (Colours are visible in the online version of the article; http://dx.doi.org/10.3233/RNN-2012120224) movement and recovery periods. A reduction in deltatheta and an enhancement in beta-gamma bands were observed during movement with respect to recovery (Table 1, Fig. 3).

\subsection{Movement of the left cybernetic hand prosthesis}

Delivery of motor commands for the phantom of the left amputated limb before the LIFEs implant recruited areas in the ipsilateral primary sensory and motor areas (left Postcentral Gyrus - BA2, BA3 and Precentral Gyrus - BA4, Fig. 2 - second row top) and bilateral premotor and supplementary motor cortex (left and right BA6, Fig. 2 - second row bottom). At this stage no contralateral primary motor cortex activity was found. The analysis of time frequency behavior evidenced activity in alpha and beta bands lasting for the whole task duration with no time-specificity.

The cerebral recruitment during the intent to move the phantom of the left amputated limb changed markedly after the four-weeks of prosthesis motor control training with implanted LIFEs. Cortical recruitment became almost symmetrical with respect to right hand movements, with selective involvement of contralateral sensorimotor cortex (right Postcentral Gyrus BA2, BA3 and Precentral Gyrus - BA4, Fig. 2 - third row). The time frequency behaviour regained evolving properties similar to those for right hand control: stronger in the first 2 seconds involving the whole alpha and beta frequencies, while weaker and more concentrated in alpha band in the $2-5 \mathrm{~s}$ period (Fig. 2 - third row right).

Before LIFE implant, the cortico-cortical coherence between the hemispheric homologous primary sensorimotor cortices (Fig. 3) was present only at low frequencies in recovery periods. During movement a reduction in all frequency bands occurred, except in the delta band where coherence was enhanced. After four weeks of training with LIFE implants communicating with the cybernetic prosthesis, a similar pattern to right hand movement was observed: although at lower levels, inter-hemispheric communication prevailed in delta and beta bands during recovery periods. During movement, a delta-theta reduction and a beta band increase relative to recovery periods were found (Table 1, Fig. 3).

The reduction in delta and enhancement in beta and gamma bands during active movement is evident both 
Table 1

Homologous hand areas' coherence. Spectral coherence between FS activity of hemispheric primary regions devoted to the hand control integrated in delta $[1,4] \mathrm{Hz}$, theta $[5,7]$, alpha $[8,12]$, beta $[13,32]$ and gamma $[33,48] \mathrm{Hz}$ bands normalized by the frequency bin numbers during motor command dispatched to each hand (Movement) and during intermingled resting periods (Recovery). The difference between the two conditions is also shown (Recovery-Movement, Diff). Band coherences are calculated integrating only significant values $(p>0.04)$

\begin{tabular}{|c|c|c|c|c|c|c|}
\hline & & Delta & Theta & Alpha & Beta & Gamma \\
\hline \multirow[t]{3}{*}{ Right hand } & Recovery & 0.1446 & 0.0530 & 0.0557 & 0.1221 & 0.0597 \\
\hline & Movement & 0.0732 & 0.0340 & 0.0476 & 0.1401 & 0.0940 \\
\hline & Diff & 0.0714 & 0.0190 & 0.0081 & -0.0180 & -0.0343 \\
\hline \multicolumn{7}{|l|}{ Left hand } \\
\hline \multirow[t]{3}{*}{ Pre } & Recovery & 0.0692 & 0.1474 & 0.1211 & 0.0778 & 0.0000 \\
\hline & Movement & 0.0984 & 0.1174 & 0.0705 & 0.0293 & 0.0000 \\
\hline & Diff & -0.0292 & 0.0300 & 0.0506 & 0.0485 & 0.0000 \\
\hline \multirow[t]{3}{*}{ Post } & Recovery & 0.1115 & 0.0877 & 0.0227 & 0.0154 & 0.0000 \\
\hline & Movement & 0.0732 & 0.0260 & 0.0000 & 0.0290 & 0.0076 \\
\hline & Diff & 0.0383 & 0.0617 & 0.0227 & -0.0136 & -0.0076 \\
\hline
\end{tabular}

for the right hand and for the POST-session of the hand cybernetic prosthesis, while an opposite pattern was observed in the pre-training session for the left phantom hand.

\section{Discussion}

\subsection{Brain plasticity due to neutrally-interfaced cybernetic hand prosthesis control}

The possibility of inducing or redirecting the organization of cortical and subcortical functional topography has been described with fMRI studies, both in acute (Maruishi et al., 2004) and in chronic EMGdriven prosthesis use (Lotze et al., 1999).

The neurophysiological processes which take place when interacting with the external world through a BMI, such as tuning of neuronal firing towards the constraints of movement, and Hebbian plasticity of neuronal networks that map the movements in cortex, are the same that underlie motor neurorehabilitation procedures (Dobkin, 2007). Thus it is of primary importance to better understand whether or not an interface is able to cause appropriate neural changes that benefit restorative plasticity. This process is facilitated by the ability of the interface to close the user-action-environment-perception-user control loop by feeding back tactile and proprioceptive sensations. Furthermore, the reduction of cognitive load required by the user to control the machine, without forcing artificial control strategies, can be achieved only through a natural interface with the muscles or nerves that originally were devoted to the lost limb. Hence, the use of a prosthesis directly interfaced with the nervous system should better compensate for lost function and avoid aberrant CNS reorganization. Nevertheless, to our knowledge, there is scant data in the literature concerning the reshaping of cerebral pathways by the use of prostheses directly coupled with the nervous system. Training for robotic hand control and for sensory perception via a multicontact peripheral nerve neural interface produced normalization of the somatosensory and motor cortical organization that had previously been rearranged following amputation-induced neurodegeneration. Our group recently described a significant reduction of the cortical area from which motor evoked potentials could be elicited in the spared muscles of affected limb (Rossini et al., 2010), and normalization of EEG desynchronization during movement preparation in the sensorimotor areas (Tombini et al., 2012). Moreover, the four weeks of training induced a clinical improvement of about $30 \%$ of PLP, an epiphenomenon of the redirection of aberrant cortical plasticity, assessed with open answer questionnaires and clinical pain scales (short form McGill - range 0-45- from 18 to 11 , Present Pain Inventory - range 0-5- from 3 to 2, Visual Analogue Scale - range 0-100\%- from $38 \%$ to $23 \%$ ), and a progressive return towards a more physiological perception of the phantom hand. The modification of cortical plasticity occurred over the same time-course as the improvement in prosthesis control performance (Rossini et al., 2010).

The present paper provides new insights on how intensive training involving the restoration of motor output and multisensory feedback (i.e., stimulation through a neural interface together with visual feedback) can induce adaptive plastic changes in the Rolandic cortices of chronic amputees. The study of 
cortical recruitment and neuronal activity patterns in our patient showed how, before training, attempting to move the phantom of the missing limb involved medial frontal gyrus and superior frontal gyrus, without activation of directly connected contralateral primary sensorimotor cortices. Such premotor and supplementary motor cortices are crucial for movement planning and coordination. It can be hypothesized that the absence of central-peripheral communication inhibits physiological engagement of execution structures, concentrating neural activity in preparatory territories (Bestmann et al., 2006; Diers et al., 2010; Raffin et al., 2012). The presence of intra-fascicular electrodes allowed new signals to be delivered through peripheral nerves towards the cortex and produced an intensive exchange of sensori-motor afferent and efferent inputs/outputs. Four weeks of training with such implanted electrodes led to a new functional recruitment of sensorimotor areas devoted to hand control, i.e., contralateral primary motor and sensory cortices (Broadmann's areas 2, 3 and 4). As expected by previous literature (Flor et al., 2001; Lotze et al., 1999), a physiologically-oriented plastic reorganization corresponded to the amelioration of clinical symptoms of PLP.

\subsection{Functional connectivity between hemispheric homologous areas characterizes motor control}

The improvement in cybernetic hand prosthesis control has been showed to be coupled with reorganization in sensorimotor cortical regions activity (Rossini et al., 2010; Tombini et al., 2012). In parallel with regional activation properties, it is well known that the motor abilities depend on intra- and inter-network functional connectivity (Hummel and Gerloff, 2006), and are anatomically related to the amount of transcallosal connections (Stancak et al., 2002). Unilateral middle cerebral artery territory stroke is a paradigmatic model of the unbalancing of primary sensorimotor areas activities (Rossini et al., 2003) together with an alteration of the associated network connectivity (Grefkes and Fink, 2011). Such connectivity has been observed to be reduced in acute severe stroke both in animal models (van Meer et al., 2010) and in humans (Carter et al., 2010), while its recovery pairs with a progressive improvement of motor control. Observing longitudinally ten patients along one year and analyzing fMRI BOLD signals during resting state, Wang et colleagues (2010) showed that the inter-hemispheric coupling between homologous primary motor cortices recovered in parallel with motor skills (Wang et al., 2010).

Neuronal oscillations of EEG in specific frequency bands play a specific functional role (delta theta, alpha beta gamma): an increase of low beta inter-hemispheric coherence between sensory-motor regions was observed in stroke patients reacquiring paretic hand control (Gerloff et al., 2006).

In our patient we cannot describe a global reproducible trend of spectral modification in single bands, but more a global behavior of spectral profile between task-oriented coherence and recovery (reduction in theta-delta band and enhancement in beta and gamma bands), that - in parallel with improvement of prosthesis control performance and PLP amelioration- became similar to the one observed with the contralateral unaffected hand and thus probably more physiological.

\subsection{FSS allows functional connectivity estimation both during movement and in resting state}

FSS allows the analysis of the identified cerebral sources in experimental conditions that differ from those in which they are identified and thus enabled us to track their activity even while the subject was at rest.

In the present study brain connectivity between the cortical areas devoted to the intact and missing hand movement was investigated during the delivering of hand movement command and in resting state. Recent evidence indicates that networks devoted to specific functional domains maintain their typical functional properties also in the resting state (Deco and Corbetta, 2011), so resting state features have been suggested to be useful for assessing the health of brain networks (Carter et al., 2010).

The analysis of coherence during the resting state allows removal of different task-related neural engagements (i.e., attention and monitoring in planning and executing the motor task) that are not comparable between patients and controls and between hemispheres in amputees. In this respect the use of FSS to evaluate functional connectivity in the resting state for specific regions devoted to the hand sensorimotor control independent of their location was extremely helpful.

Since functional sources are identified on the basis of their specific behavior during the task of 
interest and not of their topography, they can be suitably investigated even if the sensorimotor control areas have been displaced due to plastic reorganizations. Interestingly, we found that training to control the LIFE connected cybernetic prosthesis modulated the inter-hemispheric connectivity not only during movement, but also in the resting state. Conceivably in our case, rearrangements of excitatory-inhibitory local and interhemispheric balances subtend the modulation of functional connectivity between affected and unaffected sensory hand areas.

The involvement of beta and gamma-band band connectivity in sensory motor integration plays a role for the dynamic construction of neuronal networks related to sensorimotor integration (Engel and Fries, 2010; Tecchio et al., 2007a; Tecchio et al., 2008; Tombini et al., 2009). A further advantage of the FSS method is to better resolve such high frequency band rhythms in EEG scalp activity.

\section{Conclusion}

Taken together, the results of this study confirm the hypothesis that neural interfaces, such as the bidirectional interface we adopted, being able to assure a high level of interaction among the user and the cybernetic prosthesis and to transmit back sensory input from the periphery, are optimal candidates for hand prosthesis control. This is due to their ability to establish communication channels needed for a natural control of the effector designed for functional replacement. Furthermore neural interfaces recreate the connection with the environment that allow, through a continual exercise with feedback and reward, to redirect cortical orphan areas toward restorative neuroplasticity. Part of this reorganization involves bi-hemispheric networks that regain a more physiological communication necessary for motor control.

\section{Acknowledgments}

This study was partially supported by the Commission of the European Community Seventh Framework Program under grant agreement no 200859, project MEGMRI, and TIME (Contract No.: FP7-ICT-224012 - Transverse, Intrafascicular Multichannel Electrode system for induction of sensation and treatment of phantom limb pain in amputees) and by the Royal
Society International Joint Project - 2010/R1: 'The Key Movement Controllers: an EEG/fMRI study of the hand network dynamics [KeyMoCo]'. The authors deeply thank the volunteer subject of this study, who donated his time and consented to participate in all phases of the present protocol. The authors are also grateful to Andrew Jackson for his valuable comments and for editing the revised version of this manuscript.

\section{References}

Barbati, G., Porcaro, C., Hadjipapas, A., Adjamian, P., Pizzella, V., Romani, G.L., Seri, S., Tecchio, F. \& Barnes, G.R. (2008). Functional source separation applied to induced visual gamma activity. Hum Brain Mapp, 29(2), 131-141.

Barbati, G., Porcaro, C., Zappasodi, F., Rossini, P.M. \& Tecchio, F. (2004). Optimization of an independent component analysis approach for artifact identification and removal in magnetoencephalographic signals. Clin Neurophysiol, 115(5), 1220-1232.

Barbati, G., Sigismondi, R., Zappasodi, F., Porcaro, C., Graziadio, S., Valente, G., Balsi, M., Rossini, P.M. \& Tecchio, F. (2006). Functional source separation from magnetoencephalographic signals. Hum Brain Mapp, 27(12), 925-934.

Bestmann, S., Oliviero, A., Voss, M., Dechent, P., Lopez-Dolado, E., Driver, J. \& Baudewig, J. (2006). Cortical correlates of TMSinduced phantom hand movements revealed with concurrent TMS-fMRI. Neuropsychologia, 44(14), 2959-2971.

Carrozza, M.C., Cappiello, G., Micera, S., Edin, B.B., Beccai, L. \& Cipriani, C. (2006). Design of a cybernetic hand for perception and action. Biol Cybern, 95(6), 629-644.

Carter, A.R., Astafiev, S.V., Lang, C.E., Connor, L.T., Rengachary, J., Strube, M.J., Pope, D.L., Shulman, G.L. \& Corbetta, M. (2010). Resting interhemispheric functional magnetic resonance imaging connectivity predicts performance after stroke. Ann Neurol, 67(3), 365-375.

Cichocki, A. \& Amari, S.I. (2002). Adaptive Blind Signal and Image Processing. JohnWiley \& Sons, Chichester, UK.

Deco, G. \& Corbetta, M. (2011). The dynamical balance of the brain at rest. Neuroscientist, 17(1), 107-123.

Di Pino, G., Guglielmelli, E. \& Rossini, P.M. (2009). Neuroplasticity in amputees: Main implications on bidirectional interfacing of cybernetic hand prostheses. Prog Neurobiol, 88(2), 114-126.

Diers, M., Christmann, C., Koeppe, C., Ruf, M. \& Flor, H. (2010). Mirrored, imagined and executed movements differentially activate sensorimotor cortex in amputees with and without phantom limb pain. Pain, 149(2), 296-304.

Dobkin, B.H. (2007). Brain-computer interface technology as a tool to augment plasticity and outcomes for neurological rehabilitation. J Physiol, 579(Pt 3), 637-642.

Engel, A.K. \& Fries, P. (2010). Beta-band oscillations-signalling the status quo? Curr Opin Neurobiol, 20(2), 156-165.

Flor, H., Denke, C., Schaefer, M. \& Grusser, S. (2001). Effect of sensory discrimination training on cortical reorganisation and phantom limb pain. Lancet, 357(9270), 1763-1764. 
Flor, H., Elbert, T., Knecht, S., Wienbruch, C., Pantev, C., Birbaumer, N., Larbig, W. \& Taub, E. (1995). Phantom-limb pain as a perceptual correlate of cortical reorganization following arm amputation. Nature, 375(6531), 482-484.

Flor, H., Nikolajsen, L. \& Staehelin Jensen, T. (2006). Phantom limb pain: A case of maladaptive CNS plasticity? Nat Rev Neurosci, 7(11), 873-881.

Gerloff, C., Bushara, K., Sailer, A., Wassermann, E.M., Chen, R., Matsuoka, T., Waldvogel, D., Wittenberg, G.F., Ishii, K., Cohen, L.G., et al. (2006). Multimodal imaging of brain reorganization in motor areas of the contralesional hemisphere of well recovered patients after capsular stroke. Brain, 129(Pt 3), 791-808.

Gilbert S.J. \& Walsh V. (2004). Vision: The versatile 'visual' cortex. Curr Biol, 29(14), R1056-R1057. Review.

Grefkes, C. \& Fink, G.R. (2011). Reorganization of cerebral networks after stroke: New insights from neuroimaging with connectivity approaches. Brain, 134(Pt 5), 1264-1276.

Halliday, D.M., Rosenberg, J.R., Amjad, A.M., Breeze, P., Conway, B.A. \& Farmer, S.F. (1995). A framework for the analysis of mixed time series/point process data-theory and application to the study of physiological tremor, single motor unit discharges and electromyograms. Prog Biophys Mol Biol, 64(2-3), 237278.

Hoffmann, K.P. \& Kock, K.P. (2005). Final report on design consideration of tLIFE2. tech rep IBMT.

Hummel, F.C. \& Gerloff, C. (2006). Interregional long-range and short-range synchrony: A basis for complex sensorimotor processing. Prog Brain Res 159, 223-236.

Hyvarinen, A., Karhunen, J. \& Oja, E. (2001). Independent Component Analysis. JohnWiley \& Sons, Chichester, UK.

Kaas, J.H. (1991). Plasticity of sensory and motor maps in adult mammals. Annu Rev Neurosci, 14, 137-167.

Lotze, M., Grodd, W., Birbaumer, N., Erb, M., Huse, E., and Flor, $H$ (1999). Does use of a myoelectric prosthesis prevent cortical reorganization and phantom limb pain? Nat Neurosci 2(6), 501502.

Makeig, S., Debener, S., Onton, J. \& Delorme, A. (2004). Mining event-related brain dynamics. Trends Cogn Sci, 8(5), 204-210.

Maruishi, M., Tanaka, Y., Muranaka, H., Tsuji, T., Ozawa, Y., Imaizumi, S., Miyatani, M. \& Kawahara, J. (2004). Brain activation during manipulation of the myoelectric prosthetic hand: A functional magnetic resonance imaging study. Neuroimage, 21(4), 1604-1611.

Medaglia, M.T., Tecchio, F., Seri, S., Di Lorenzo, G., Rossini, P.M. \& Porcaro, C. (2009). Contradiction in universal and particular reasoning. Hum Brain Mapp, 30(12), 4187-4197.

Mercier, C., Reilly, K.T., Vargas, C.D., Aballea, A. \& Sirigu, A. (2006). Mapping phantom movement representations in the motor cortex of amputees. Brain, 129(Pt 8), 2202-2210.

Merzenich, M.M., Nelson, R.J., Stryker, M.P., Cynader, M.S., Schoppmann, A. \& Zook, J.M. (1984). Somatosensory cortical map changes following digit amputation in adult monkeys. J Comp Neurol, 224(4), 591-605.

Micera, S., carrozza, M., Beccai, L., Vecchi, F. \& Dario, P. (2006). Hybrid bionic systems for the replacement of hand function. IEEE Proceedings, 94(9), 1752-1762.
Micera, S. \& Navarro, X. (2009). Bidirectional interfaces with the peripheral nervous system. Int Rev Neurobiol, 86, 23-38.

Micera, S., Navarro, X., Carpaneto, J., Citi, L., Tonet, O., Rossini, P.M., Carrozza, M.C., Hoffmann, K.P., Vivo, M., Yoshida, K. et al. (2008). On the use of longitudinal intrafascicular peripheral interfaces for the control of cybernetic hand prostheses in amputees. IEEE Trans Neural Syst Rehabil Eng, 16(5), 453472.

Navarro, X., Krueger, T.B., Lago, N., Micera, S., Stieglitz, T. \& Dario, P. (2005). A critical review of interfaces with the peripheral nervous system for the control of neuroprostheses and hybrid bionic systems. J Peripher Nerv Syst, 10(3), 229-258.

Pascual-Leone, A., Amedi, A., Fregni, F. \& Merabet LB. (2005). The plastic human brain cortex. Annu Rev Neurosci, 28, 377-401. Review.

Pascual-Marqui, R.D. (2002). Standardized low-resolution brain electromagnetic tomography (sLORETA): Technical details. Methods Find Exp Clin Pharmacol, 24 (Suppl D), 5-12.

Pfurtscheller, G. \& Lopes da Silva, F.H. (1999). Event-related EEG/MEG synchronization and desynchronization: Basic principles. Clin Neurophysiol, 110(11), 1842-1857.

Pons, T.P., Garraghty, P.E., Ommaya, A.K., Kaas, J.H., Taub, E. \& Mishkin, M. (1991). Massive cortical reorganization after sensory deafferentation in adult macaques. Science, 252(5014), 1857-1860.

Porcaro, C., Barbati, G., Zappasodi, F., Rossini, P.M. \& Tecchio, F. (2008). Hand sensory-motor cortical network assessed by functional source separation. Hum Brain Mapp, 29(1), $70-81$.

Porcaro, C., Coppola, G., Di Lorenzo, G., Zappasodi, F., Siracusano, A., Pierelli, F., Rossini, P.M., Tecchio, F. \& Seri, S. (2009). Hand somatosensory subcortical and cortical sources assessed by functional source separation: An EEG study. Hum Brain Mapp, 30(2), 660-674.

Porcaro, C., Ostwald, D. \& Bagshaw, A.P. (2010). Functional source separation improves the quality of single trial visual evoked potentials recorded during concurrent EEG-fMRI. Neuroimage, $50(1), 112-123$.

Porcaro, C., Ostwald, D., Hadjipapas, A., Barnes, G.R. \& Bagshaw, A.P. (2011). The relationship between the visual evoked potential and the gamma band investigated by blind and semi-blind methods. Neuroimage, 56(3), 1059-1071.

Porcaro, C., Zappasodi, F., Barbati, G., Salustri, C., Pizzella, V., Rossini, P.M. \& Tecchio, F. (2006). Fetal auditory responses to external sounds and mother's heart beat: Detection improved by Independent Component Analysis. Brain Res, 1101(1), 51-58.

Raffin, E., Giraux, P. \& Reilly, K.T. (2011). The moving phantom: Motor execution or motor imagery? Cortex 48(6), 746-757.

Raffin, E., Mattout, J., Reilly, K.T. \& Giraux, P. (2012). Disentangling motor execution from motor imagery with the phantom limb. Brain, 135(Pt 2), 582-595.

Ramachandran, V.S., Rogers-Ramachandran, D. \& Stewart, M. (1992). Perceptual correlates of massive cortical reorganization. Science, 258(5085), 1159-1160. 
Reilly, K.T., Mercier, C., Schieber, M.H. \& Sirigu, A. (2006). Persistent hand motor commands in the amputees' brain. Brain, 129(Pt 8), 2211-2223.

Rossini, P.M., Calautti, C., Pauri, F. \& Baron, J.C. (2003). Poststroke plastic reorganisation in the adult brain. Lancet Neurol, 2(8), 493-502.

Rossini, P.M., Micera, S., Benvenuto, A., Carpaneto, J., Cavallo, G., Citi, L., Cipriani, C., Denaro, L., Denaro, V., Di Pino, G., et al. (2010). Double nerve intraneural interface implant on a human amputee for robotic hand control. Clin Neurophysiol, 121(5), 777-783.

Sadato, N. (2005). How the blind "see" Braille: Lessons from functional magnetic resonance imaging. Neuroscientist, 11, 577-582. Review.

Stancak, A., Lucking, C.H. \& Kristeva-Feige, R. (2002). The size of corpus callosum and functional connectivities of cortical regions in finger and shoulder movements. Brain Res Cogn Brain Res, 13(1), 61-74.

Tecchio, F., Graziadio, S., Barbati, G., Sigismondi, R., Zappasodi, F., Porcaro, C., Valente, G., Balsi, M. \& Rossini, P.M. (2007a). Somatosensory dynamic gamma-band synchrony: A neural code of sensorimotor dexterity. Neuroimage, 35(1), 185-193.

Tecchio, F., Porcaro, C., Barbati, G. \& Zappasodi, F. (2007b). Functional source separation and hand cortical representation for a brain-computer interface feature extraction. J Physiol, 580(Pt 3), 703-721.

Tecchio, F., Zappasodi, F., Porcaro, C., Barbati, G., Assenza, G., Salustri, C. \& Rossini, P.M. (2008). High-gamma band activity of primary hand cortical areas: A sensorimotor feedback efficiency index. Neuroimage, 40(1), 256-264.

Tombini, M., Rigosa, J., Zappasodi, F., Porcaro, C., Citi, L., Carpaneto, J., Rossini, P.M. \& Micera, S. (2012). Combined analysis of cortical (EEG) and nerve stump signals improves robotic hand control. Neurorehabil Neural Repair, 26(3), 275281.

Tombini, M., Zappasodi, F., Zollo, L., Pellegrino, G., Cavallo, G. Tecchio, F., Guglielmelli, E. \& Rossini, P.M. (2009). Brain activity preceding a $2 \mathrm{D}$ manual catching task. Neuroimage, 47(4), 1735-1746.

van Meer, M.P., van der Marel, K., Wang, K., Otte, W.M., El Bouazati, S., Roeling, T.A., Viergever, M.A., Berkelbach van der Sprenkel, J.W. \& Dijkhuizen, R.M. (2010). Recovery of sensorimotor function after experimental stroke correlates with restoration of resting-state interhemispheric functional connectivity. J Neurosci, 30(11), 3964-3972.

Wang, L., Yu, C., Chen, H., Qin, W., He, Y., Fan, F., Zhang, Y., Wang, M., Li, K., Zang, Y., et al. (2010). Dynamic functional reorganization of the motor execution network after stroke. Brain, 133(Pt 4), 1224-1238.

Zollo, L., Roccella, S., Guglielmelli, E., Carrozza, M.C. \& Dario, P. (2007). Biomechatronic design and control of an anthropomorphic artificial hand for prosthetic and robotic applications. Paper presented at: IEEE/ASME Transactions on Mechatronics, 12(4), 418-429. 